\title{
Use of methylation profiling to identify significant differentially methylated genes in bone marrow mesenchymal stromal cells from acute myeloid leukemia
}

\author{
JING HUANG ${ }^{1,2^{*}}$, ZHI LIU ${ }^{1 *}$, YUFAN SUN ${ }^{2}$, QI ZHONG ${ }^{1}$, LI XU² ${ }^{2}$, RUIMIN OU ${ }^{1}$, CHENG LI $^{1}$, \\ RUI CHEN ${ }^{1}$, MENGDONG YAO ${ }^{1}$, QING ZHANG $^{1}$ and SHUANG LIU ${ }^{1}$ \\ ${ }^{1}$ Department of Hematology, Guangdong Second Provincial General Hospital, Guangzhou, Guangdong 510317; \\ ${ }^{2}$ Department of Hematology, The First Hospital of Kashgar, Kashgar, Xinjiang 844000, P.R. China
}

Received September 13, 2016; Accepted October 19, 2017

DOI: 10.3892/ijmm.2017.3271

\begin{abstract}
The present study aimed to characterize the epigenetic architecture by studying the DNA methylation signature in bone marrow mesenchymal stem cells (BM-MSCs) from patients with acute myeloid leukemia (AML). Microarray dataset GSE79695 was downloaded from the Gene Expression Omnibus database. Differentially methylated sites and differentially methylated $\mathrm{CpG}$ islands were identified in BM-MSC samples from patients with AML compared with controls. MicroRNAs (miRs) encoding genes covering differentially methylated sites were found and the regulation network was constructed. Pathway enrichment analysis of hypermethylated genes and hypomethylated genes was performed, followed by protein-protein interaction (PPI) network construction. Moreover, the identified differentially methylated genes were compared with the leukemia-related marker/therapeutic genes from the literature. Overall, 228 hypermethylated CpG site probes covering 183 gene symbols and 523 hypomethylated $\mathrm{CpG}$ sites probes covering 362 gene symbols were identified in the BM-MSCs from AML patients. Furthermore, 4 genes with $\mathrm{CpG}$ island hypermethylation were identified, including peptidase M20 domain containing 1 (PM20D1). The hsa-miR-596-encoding gene MIR596 was found to be hypermethylated and the regulation network based on hsa-miR-596 and its targets (such as cytochrome P450 family 1 subfamily B member 1) was constructed. Hypermethylated and hypomethylated genes were enriched in different Kyoto Encyclopedia of Genes and Genomes pathways, including 'hsa05221: Acute myeloid leukemia' and 'hsa05220: Chronic myeloid leukemia',
\end{abstract}

Correspondence to: Dr Qing Zhang or Dr Shuang Liu, Department of Hematology, Guangdong Second Provincial General Hospital, 466 Xin Gang Zhong Road, Guangzhou, Guangdong 510317, P.R. China E-mail: zhqing@vip.163.com

E-mail: shuangliu249@hotmail.com

${ }^{*}$ Co-first authors

Key words: acute myeloid leukemia, bone marrow mesenchymal stem cells, differentially methylated $\mathrm{CpG}$ islands, microRNA which the hypomethylated gene mitogen-activated protein kinase 3 (MAPK3) was involved in. In addition, $M A P K 3$, lysine demethylase 2B and RAP1A, member of RAS oncogene family were hubs in the PPI network of methylated genes. In conclusion, PM20D1 with hypermethylation of $\mathrm{CpG}$ islands may be associated with the energy expenditure of patients with AML. Furthermore, the aberrantly hypermethylated miR-159-encoding gene MIR159 may be a potential biomarker of AML.

\section{Introduction}

Acute myeloid leukemia (AML) is an aggressive, genetically heterogeneous malignancy characterized by the accumulation of abnormal blasts in the bone marrow (BM) (1). These hematopoietic progenitor cells interfere with normal hematopoiesis, can escape into the peripheral blood, and infiltrate the lungs and central nervous system (2). AML can occur in individuals of all ages, but evidence has shown that AML is most common in older patients ( $>65$ years) (3) and that it accounts for $70 \%$ of acute leukemia cases in adults (4). Current treatments include intensive chemotherapy and BM transplantation (5). Although there have been large improvements in outcome in recent decades, AML remains a life-threatening malignancy, with an 5-year overall survival rate of only $40-45 \%$ in young patients and $>10 \%$ in the elderly, due to either resistance to therapy or relapsed AML (1). Thus, gaining a better understanding of the underlying molecular mechanism of AML is imperative for the development of more valuable diagnostic and therapeutic targets.

Molecular analyses have expanded our understanding of the heterogeneity of AML, which leads to distinct clinical presentations in different cytogenetic and morphological subtypes (6). It is clear that genetic events [such as point mutations, and insertions and deletions (indels)] and recurrent chromosomal abnormalities (such as numeric abnormalities and translocations) are necessary for the development of AML (7). For instance, genomic and functional studies have demonstrated two broad classes of mutations that collaborate to cause AML when neither is sufficient to do so in isolation $(1,8)$. Class I mutations, such as neuroblastoma RAS viral oncogene homolog mutations, confer a proliferative advantage of hematopoietic stem and progenitor cells (HSPCs), but have no effect on differentiation. However, class II mutations, 
such as translocations involving the mixed lineage leukemia 1 gene, block the differentiation of HSPCs and subsequent apoptosis (1). On the other hand, deregulated epigenetic changes have been shown as major components of the pathogenesis of AML (7). DNA methylation signatures have been used to identify distinct epigenetically defined subtypes in AML (9). Akalin et al demonstrated that aberrant DNA methylation patterns in AML were highly specific and associated with specific driving genetic lesions (10). In addition, the findings of Tao et al showed that epigenetic inactivation of microRNA (miRNA/miR)-663 by promoter hypermethylation could be found in AML cell lines and pediatric AML samples (11). However, the gene methylation signatures in AML are not completely understood. Identifying more differentially methylated genes may provide a better understanding of the pathogenesis of the disease.

BM mesenchymal stem cells (BM-MSCs) are key components of the hematopoietic microenvironment and are particularly important hematopoietic regulators due to their capacity to self-renewal and to differentiate into different stromal cell lines and produce soluble factors facilitating hematopoietic cell maintenance (12). Previously, Blau et al demonstrated that BM-MSCs from patients with myelodysplastic syndrome (MDS) and AML showed chromosomal abnormalities, suggesting potential involvement of BMSCs in the pathophysiology of MDS/AML (13). Furthermore, a distinctive gene expression profile of MSCs was identified from pediatric cases of AML compared with healthy donors (14). However, the gene methylation patterns in BM-MSC from patients with AML have not been fully addressed.

In the present study, recently deposited microarray data (deposited on March 29, 2016) from a public database were downloaded and reanalyzed to study the methylation status in BM-MSCs from patients with AML. Differentially methylated sites and differentially methylated $\mathrm{CpG}$ islands were identified in BM-MSC samples from patients with AML compared with controls. miRNA-encoding genes covering differentially methylated sites were found and the regulation network was constructed. Pathway enrichment analysis of hypermethylated genes and hypomethylated genes was performed, followed by protein-protein interaction (PPI) network construction. Moreover, the identified differentially methylated genes were compared with the leukemia-related marker/therapeutic genes from the literature. The study aimed to characterize the epigenetic architecture by studying the DNA methylation signature in BM-MSCs from AML patients. Unraveling the complexities of the methylation changes of AML has important implications for diagnosis and the development of novel targets for therapy.

\section{Materials and methods}

Microarray data and data preprocessing. The Gene Expression Omnibus (GEO) database (http://www.ncbi.nlm.nih.gov/geo/) serves as the major public repository for microarray, next-generation sequencing functional genomic data sets and other data types, such as genome methylation status analyses (15). In the present study, the methylation profiling dataset GSE79695 was downloaded from the GEO database (16). This downloaded dataset included 32 BM-MSC samples derived from patients with AML (these samples were defined as AML) and 12 BM-MSC samples from healthy donor controls (these samples were defined as the control). The GPL13534 Illumina HumanMethylation450 BeadChip (HumanMethylation450_15017482) platform (Illumina, Inc., San Diego, CA, USA) was used.

The signal intensity files of the GSE79695 dataset were downloaded. Prior to proceeding with methylation data analysis. The methylation status of the measured $\mathrm{CpG}$ sites was determined by calculation of the $\beta$-value (a value between 0 and 1) (17), with 1 indicating totally methylated and 0 representing unmethylated. The $\beta$-values are proportional to the ratio of intensities between the methylated and unmethylated alleles according to the following formula: $\beta=$ methylated signal / (methylated signal + unmethylated signal +100$)$; a constant bias of 100 was added to regularize the $\beta$-value when the methylated signal value and the unmethylated signal values were small (18).

Detection of differentially methylated sites and differentially methylated $\mathrm{CpG}$ islands. The City of Hope $\mathrm{CpG}$ Island Analysis Pipeline (COHCAP) package in Bioconductor (https://sourceforge.net/projects/cohcap/) is an algorithm to analyze either Illumina methylation array or bisulfite sequencing data, providing tools for data integration with methylated $\mathrm{CpG}$ sites and providing statistics to define differentially methylated regions (DMRs) (CpG islands) (19). COHCAP uses $\beta$-values or methylation proportions as the input (20). In the present study, following data pre-processing, differential analyses were performed to compare the differences in methylation between the AML group and the control group. $\delta-\beta$ was calculated as the difference in the mean $\beta$-values for each $\mathrm{CpG}$ site using the COHCAP package to estimate the differential methylation at each $\mathrm{CpG}$ site between the AML group and the control group. The threshold for DMRs was set as absolute value of $\delta-\beta>0.1$ and $\mathrm{P}<0.05 ; \delta-\beta>0.1$ was defined as hypermethylated sites and $\delta-\beta<-0.1$ was considered to show hypomethylated sites. The differentially methylated $\mathrm{CpG}$ sites were mapped to gene symbols.

Furthermore, $\mathrm{CpG}$ island statistics could be calculated by averaging $\beta$-values among samples per site and comparing the average $\beta$-values across groups using the COHCAP package. A minimum number of sites can be specified to define a $\mathrm{CpG}$ island according to the COHCAP CpG island analysis (19). The parameter 'num.sites' indicates the minimum number of sites within a $\mathrm{CpG}$ island. In the present study, the parameter 'num.sites' was set as 4 and the identified $\mathrm{CpG}$ islands were considered as differentially methylated $\mathrm{CpG}$ islands. The other thresholds were set the same as for differentially methylated $\mathrm{CpG}$ site screening.

Detection of differentially methylated miRNA encoding genes and construction of regulation network. From the identified differentially methylated $\mathrm{CpG}$ sites in the aforementioned method, the miRNA encoding genes containing differentially methylated $\mathrm{CpG}$ sites were screened. miRNA target predictions for the differentially methylated miRNA-encoding genes were conducted using the miRWalk2 online tool (http://zmf.umm.uni-heidelberg.de/apps/zmf/mirwalk2), a comprehensive atlas of validated and predicted miRNA-target interactions (21). The 'predicted target module' was used and genes with SUM = 4 [miRWalk, miRanda (http://www. microrna.org), RNA22 (http://cm.jefferson.edu/rna22/) and TargetScan (http://www.targetscan.org/)] were denoted as 
A

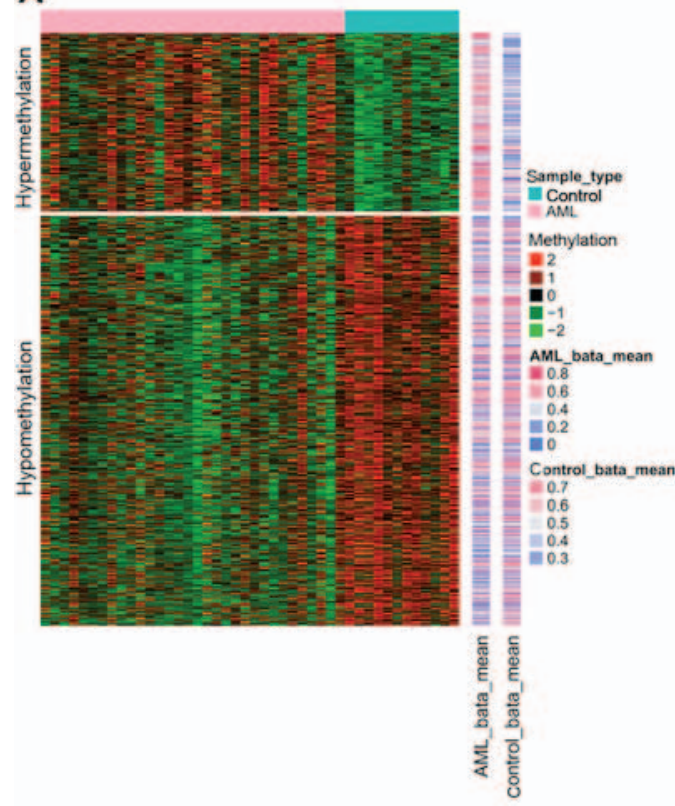

B

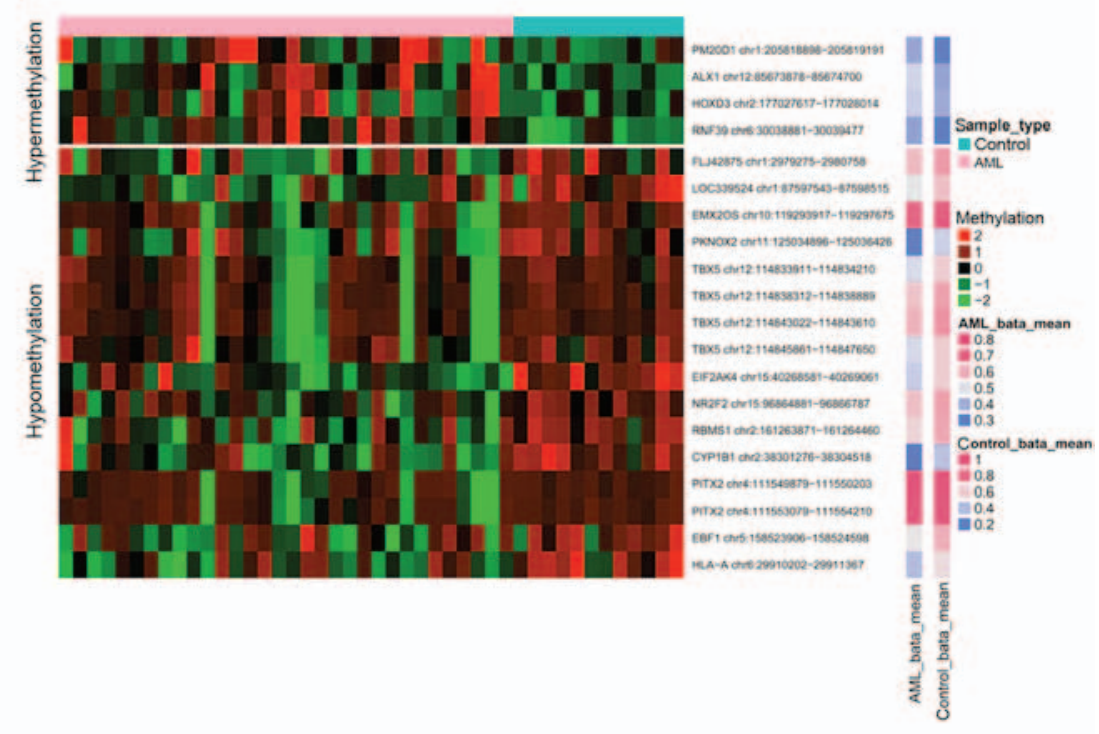

Figure 1. Heat map of DMRs and differentially methylated CpG islands. (A) Heat map of DMRs. Rows indicate different samples, while columns represent CpG site probes. (B) Heat map of differentially methylated CpG islands. DMRs, differentially methylated regions; AML, acute myeloid leukemia.

target genes for miRNA. Next, these genes were compared with the identified DMR-related genes, and only the overlapping genes were used for regulation network construction using Cytoscape version 3.2.0 (22).

Pathway enrichment analysis. The Database for Annotation, Visualization and Integrated Discovery (DAVID) bioinformatics resource is a web-accessible gene database, consisting of an integrated biological knowledge base and novel algorithms to systematically extract biological meaning from large gene/protein lists (23).

In the present study, to further investigate the functional effects of DNA methylation in BM-MSCs of AML, Kyoto Encyclopedia of Genes and Genomes (KEGG) pathway enrichment analysis (http://www.genome.jp/kegg/) of hypermethylated and hypomethylated genes was conducted using DAVID (version 6.8). Analyses were grounded on a hypergeometric test with a P-value of $<0.05$. Pathways enriched by at least 2 DMR-related genes were enriched.

Construction of PPI network for DMR-related genes. The Search Tool for the Retrieval of Interacting Genes (STRING) online database resource provides a critical integration of protein-protein interactions, including experimental and predicted interaction information with a confidence score (24). Direct (physical) as well as indirect (functional) associations are scored and integrated, resulting in comprehensive protein networks, in which proteins are represented with nodes and interactions between any two proteins are shown with an edge (24). In the present study, the PPI network was constructed using STRING (version 10.0) (24) and visualized using Cytoscape (25), a network visualization and analysis software. The gene inputs were all the identified aberrantly methylated genes in the AML group versus the control group, and the species was chosen as Homo sapiens. Finally, the degree of nodes (proteins) in the PPI network was calculated and the nodes with a higher degree were deemed to be hubs in the network.

Analysis of leukemia-associated differentially methylated genes. The Comparative Toxicogenomics Database (CTD; http://ctdbase.org/) is a unique scientific resource that can provide detailed information on gene-disease relationships, chemical-gene interactions and chemical-disease relationships, which may predict numerous novel associations between different data types (26). In the present study, gene-leukemia associations of 'marker' or 'therapeutic' type (http://ctd.mdibl.org/help/glossary.jsp for description of the original labels) were parsed (27). The term 'marker' referred to a gene that may be a biomarker of a disease (e.g., increased expression of gene $\mathrm{X}$ correlates with leukemia) or play a role in the etiology of a disease (e.g., mutations in gene $X$ cause leukemia). The term 'therapeutic' referred to a gene that is or may be a therapeutic target in the treatment a disease (e.g., targeted reduction of gene $\mathrm{X}$ expression reduces susceptibility to leukemia). In the present study, the marker/therapeutic genes associated with leukemia were all downloaded and were combined with the identified methylated genes to identify the leukemia-associated differentially methylated genes.

\section{Results}

Identification of differentially methylated $\mathrm{Cp} G$ sites and differentially methylated $\mathrm{CpG}$ islands. Out of the total $\mathrm{CpG}$ islands analyzed, only 751 probes containing differentially methylated CpG sites were found. Overall, 228 hypermethylated $\mathrm{CpG}$ site probes corresponded to 183 gene symbols and 523 hypomethylated $\mathrm{CpG}$ site probes were proportional to 362 gene symbols. The heat map of differentially methylated $\mathrm{CpG}$ sites is presented in Fig. 1A. 
Table I. Statistical information of differentially methylated CpG islands.

\begin{tabular}{|c|c|c|c|c|c|}
\hline Island & Gene & AML.avg. $\beta$ & Control.avg. $\beta$ & 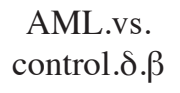 & Island.P-value \\
\hline chr6:30038881-30039477 & RNF39 & 0.375033 & 0.231256 & 0.143776 & $1.01 \times 10^{-4}$ \\
\hline chr12:85673878-85674700 & $A L X 1$ & 0.475953 & 0.337848 & 0.138105 & $6.76 \times 10^{-4}$ \\
\hline chr1:205818898-205819191 & PM20D1 & 0.384421 & 0.24664 & 0.137781 & $1.63 \times 10^{-2}$ \\
\hline chr2:177027617-177028014 & $H O X D 3$ & 0.462079 & 0.356489 & 0.10559 & $1.36 \times 10^{-2}$ \\
\hline chr4:111549879-111550203 & PITX2 & 0.765334 & 0.88268 & -0.11735 & $5.27 \times 10^{-3}$ \\
\hline chr12:114833911-114834210 & $T B X 5$ & 0.487159 & 0.604552 & -0.11739 & $5.94 \times 10^{-3}$ \\
\hline chr4:111553079-111554210 & PITX2 & 0.704864 & 0.823422 & -0.11856 & $2.95 \times 10^{-3}$ \\
\hline chr12:114845861-114847650 & $T B X 5$ & 0.48455 & 0.603446 & -0.1189 & $6.23 \times 10^{-3}$ \\
\hline $\operatorname{chr} 1: 87597543-87598515$ & LOC 339524 & 0.50858 & 0.629317 & -0.12074 & $3.65 \times 10^{-3}$ \\
\hline chr15:96864881-96866787 & $N R 2 F 2$ & 0.576133 & 0.697931 & -0.1218 & $6.74 \times 10^{-4}$ \\
\hline chr10:119293917-119297675 & EMX2OS & 0.692846 & 0.816344 & -0.1235 & $4.85 \times 10^{-3}$ \\
\hline chr1:2979275-2980758 & FLJ42875 & 0.58233 & 0.708994 & -0.12666 & $2.30 \times 10^{-2}$ \\
\hline chr12:114838312-114838889 & $T B X 5$ & 0.569495 & 0.696655 & -0.12716 & $2.91 \times 10^{-3}$ \\
\hline chr12:114843022-114843610 & $T B X 5$ & 0.594515 & 0.724575 & -0.13006 & $2.38 \times 10^{-3}$ \\
\hline chr6:29910202-29911367 & $H L A-A$ & 0.427433 & 0.566239 & -0.13881 & $5.48 \times 10^{-4}$ \\
\hline chr2:161263871-161264460 & RBMS1 & 0.543212 & 0.683953 & -0.14074 & $4.80 \times 10^{-4}$ \\
\hline chr15:40268581-40269061 & $E I F 2 A K 4$ & 0.458752 & 0.600674 & -0.14192 & $1.06 \times 10^{-5}$ \\
\hline chr2:38301276-38304518 & $C Y P 1 B 1$ & 0.283591 & 0.425883 & -0.14229 & $3.96 \times 10^{-5}$ \\
\hline chr11:125034896-125036426 & $P K N O X 2$ & 0.320788 & 0.464314 & -0.14353 & $1.59 \times 10^{-3}$ \\
\hline chr5:158523906-158524598 & $E B F 1$ & 0.517857 & 0.66552 & -0.14766 & $1.19 \times 10^{-2}$ \\
\hline
\end{tabular}

AML, acute myeloid leukemia.

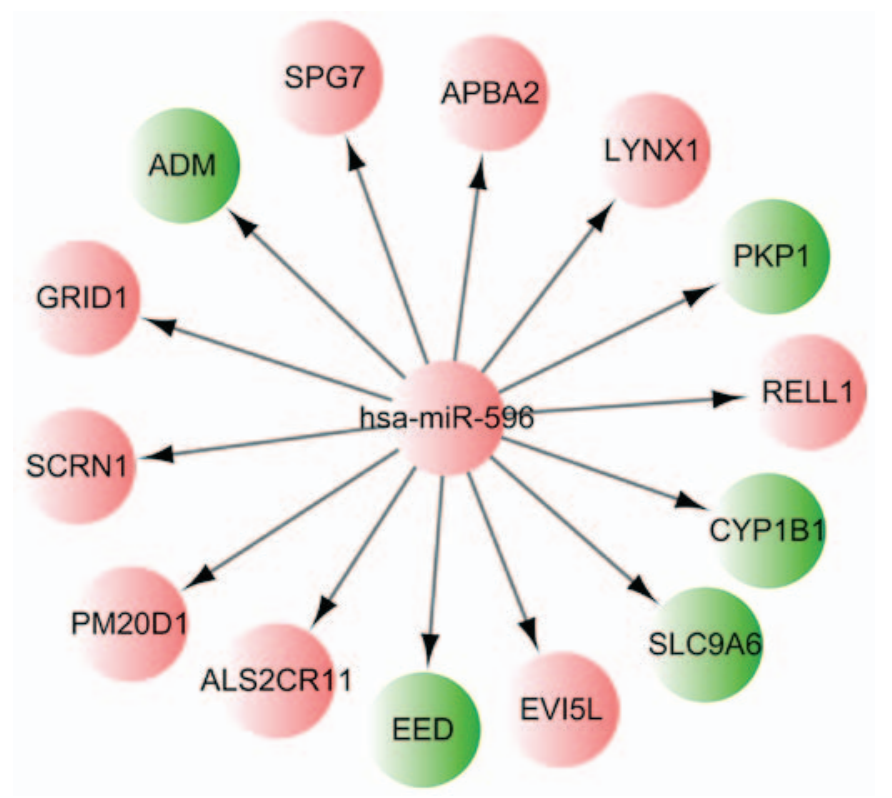

Figure 2. Regulation network based on the hsa-miR-596 and differentially methylated target genes. Red nodes indicate hypermethylated genes and green nodes represent hypomethylated genes. miR, microRNA.

On the other hand, a total of 20 differentially methylated $\mathrm{CpG}$ islands covering 16 genes were screened out, including 4 hypermethylated $\mathrm{CpG}$ islands and 16 hypomethylated $\mathrm{CpG}$ islands. The 4 genes with hypermethylated $\mathrm{CpG}$ islands were ring finger protein 39, ALX homeobox 1, peptidase M20 domain containing 1 (PM20D1) and homeobox D3. The detailed information on these differentially methylated $\mathrm{CpG}$ islands is summarized in Table I. These 16 genes containing differentially methylated $\mathrm{CpG}$ islands were DMR-related genes. The heat map of differentially methylated $\mathrm{CpG}$ islands is presented in Fig. 1B.

Identification and analysis of differentially methylated miRNA-encoding genes. Among the identified 751 differentially methylated CpG sites, the hsa-miR-596 encoding gene MIR596 was found to be hypermethylated. The corresponding CpG site of MIR596 was cg09899173, located in chr8:1764328-1765171. Using the miRWalk2 tool and the identified DMR-related genes, it was found that 14 target genes of hsa-miR-596 were differentially methylated in the MSC samples of the AML patients, including PM20D1 and cytochrome P450 family 1 subfamily B member 1 ( $C Y P 1 B 1$ ). The regulation network based on hsa-miR-596 and those 14 differentially methylated target genes is presented in Fig. 2.

Pathway enrichment analysis of genes covering differentially methylated loci. To further address the associated functional changes of gene methylation, KEGG enrichment analysis was performed. The results showed that the hypermethylated genes, adenylate cyclase 1 (Brain), ribosomal protein S6 kinase A2 (RPS6KA2), glutamate ionotropic receptor NMDA type subunit 2A and RAP1A, member of RAS oncogene family $(R A P 1 A)$, were enriched in only one KEGG pathway, 'hsa04720: Long-term potentiation'. By contrast, the hypomethylated genes were enriched in 18 KEGG pathways (Fig. 3). 
Table II. Methylation information of the genes enriched in 'acute myeloid leukemia' and 'chronic myeloid leukemia' pathways.

\begin{tabular}{|c|c|c|c|c|c|c|c|}
\hline CpG site & Chr & Loc & Gene & AML.avg. $\beta$ & Control.avg. $\beta$ & AML.vs.control.o. $\beta$ & $\begin{array}{l}\text { AML.vs.control. } \\
\text { P-value }\end{array}$ \\
\hline $\operatorname{cg} 00300298$ & 20 & 30308956 & $B C L 2 L 1$ & 0.30464 & 0.406081 & -0.10144 & $6.61 \times 10^{-4}$ \\
\hline $\operatorname{cg} 02286008$ & 16 & 30133247 & MAPK3 & 0.441586 & 0.547321 & -0.10574 & $2.26 \times 10^{-2}$ \\
\hline $\operatorname{cg} 02823329$ & 17 & 8792092 & PIK3R5 & 0.453838 & 0.560743 & -0.1069 & $1.18 \times 10^{-2}$ \\
\hline $\operatorname{cg} 13274938$ & 17 & 38493822 & $R A R A$ & 0.604901 & 0.715056 & -0.11016 & $8.96 \times 10^{-5}$ \\
\hline $\operatorname{cg} 05902503$ & 16 & 30133175 & МAPK3 & 0.465769 & 0.578058 & -0.11229 & $6.49 \times 10^{-3}$ \\
\hline $\operatorname{cg} 21893559$ & 19 & 4121075 & $M A P 2 K 2$ & 0.345042 & 0.460143 & -0.1151 & $2.38 \times 10^{-3}$ \\
\hline $\operatorname{cg} 13368805$ & $\mathrm{X}$ & 47420179 & $A R A F$ & 0.165181 & 0.2926 & -0.12742 & $3.24 \times 10^{-2}$ \\
\hline
\end{tabular}

AML, acute myeloid leukemia; Chr, chromosome; Loc, location.

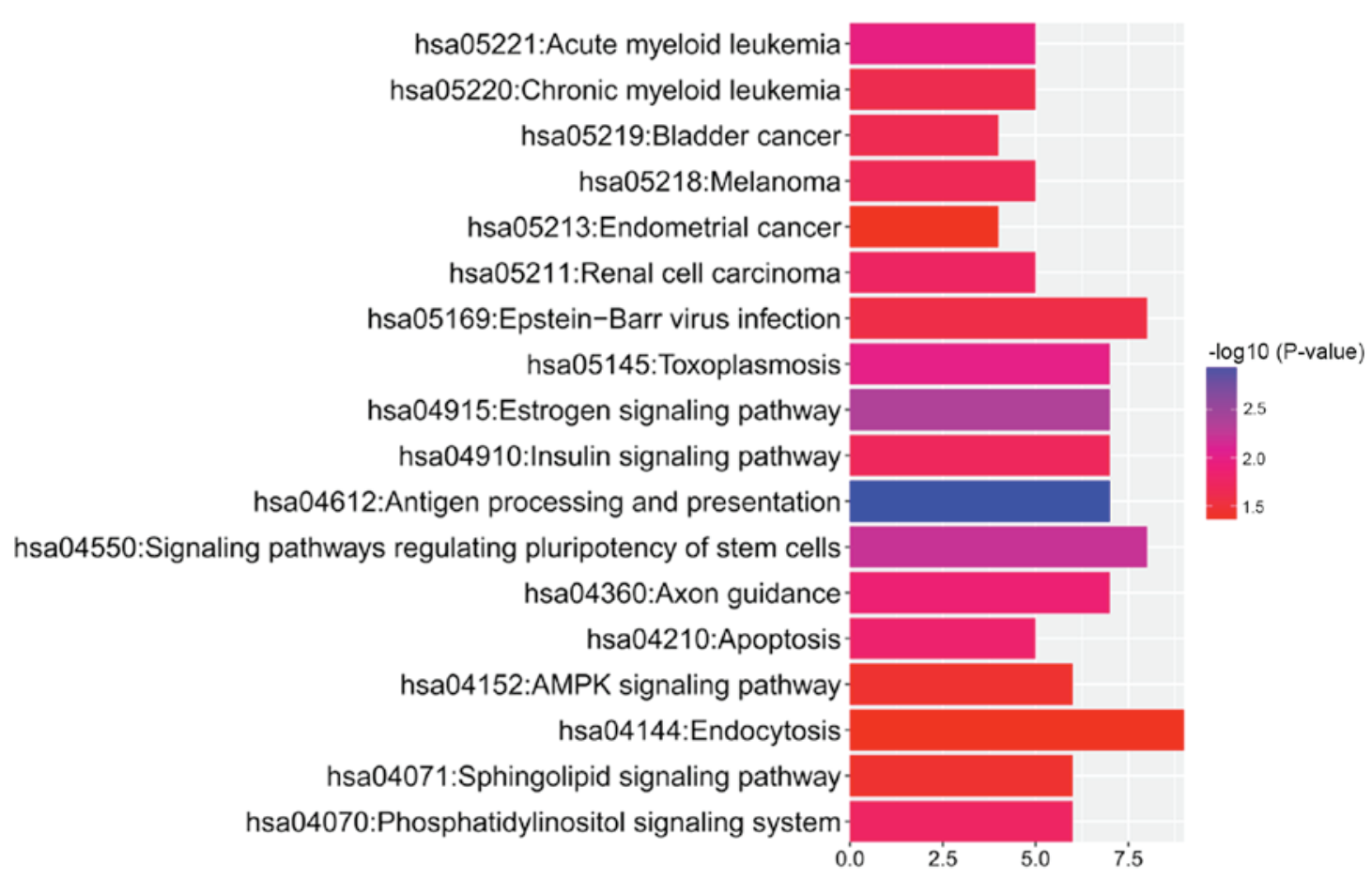

Figure 3. Kyoto Encyclopedia of Genes and Genomes pathways for the hypomethylated genes.

Moreover, pathway analysis revealed that the hypomethylated genes could be enriched in 'hsa05221: Acute myeloid leukemia' [related hypomethylated genes: Mitogen-activated protein kinase kinase 2 (MAP2K2), mitogen-activated protein kinase 3 (MAPK3), A-Raf proto-oncogene, serine/threonine kinase $(A R A F)$, retinoic acid receptor $\alpha(R A R A)$ and phosphoinositide-3-kinase regulatory subunit 5 (PIK3R5)] and 'hsa05220: Chronic myeloid leukemia' [related hypomethylated genes: MAP2K2, MAPK3, ARAF, PIK3R5 and BCL2 like $1(B C L 2 L 1)]$. The detailed methylation information of the hypomethylated genes enriched in 'acute myeloid leukemia' and 'chronic myeloid leukemia' pathways is presented in Table II.

PPI network construction and analysis. According to the PPI data from the STRING database, the resulting PPI network of aberrantly methylated genes consisted of 349 nodes (proteins) and 633 interactions (edges) (Fig. 4). The top 10 nodes with the highest degree of connectivity in the PPI network were lysine demethylase 2B (KDM2B; degree=21), MAPK3 (degree=19), RAPlA (degree=19), dihydropyrimidine dehydrogenase $(D P Y D$; degree=18), Forkhead box A2 (degree=17), ISL LIM homeobox 1 (degree=16), GATA binding protein 2 (degree=16), paired box 3 (degree=15), discs large homolog 3 (degree=15) and RPS6KA3 (degree=13). Based on the results of pathway enrichment analysis, the hub gene MAPK3 (degree $=19$ ) was also found to be a gene that was enriched in the 'hsa05221: Acute myeloid leukemia' and 'hsa05220: Chronic myeloid leukemia' pathways. Other genes with a degree of connectivity in these 2 pathways were $M A P 2 K 2$ (degree=9), $A R A F$ (degree=9), $R A R A$ (degree=9), PIK3R5 (degree=3) and $B C L 2 L 1$ (degree=8).

Analysis of leukemia-associated differentially methylated genes. A total of 337 leukemia-related marker/therapeutic 
Table III. Detailed information of 11 leukemia-associated differentially methylated genes.

\begin{tabular}{|c|c|c|c|c|c|c|c|}
\hline CpG site & $\mathrm{Chr}$ & Loc & Gene & AML.avg. $\beta$ & Control.avg. $\beta$ & $\begin{array}{l}\text { AML.vs. } \\
\text { control. } \delta . \beta\end{array}$ & $\begin{array}{c}\text { AML.vs.control. } \\
\text { P-value }\end{array}$ \\
\hline $\operatorname{cg} 22454769$ & 2 & 106000000 & FHL2 & 0.511226 & 0.381313 & 0.129913 & $4.21 \times 10^{-2}$ \\
\hline $\operatorname{cg} 24348495$ & 20 & 62693971 & TCEA2 & 0.403689 & 0.283169 & 0.12052 & $9.24 \times 10^{-5}$ \\
\hline $\operatorname{cg} 24598973$ & 1 & 969825 & AGRN & 0.444034 & 0.54644 & -0.10241 & $8.47 \times 10^{-3}$ \\
\hline $\operatorname{cg} 12944530$ & 2 & 202000000 & CFLAR & 0.209894 & 0.312596 & -0.1027 & $1.18 \times 10^{-2}$ \\
\hline $\operatorname{cg} 11354105$ & 2 & 25475805 & DNMT3A & 0.42197 & 0.526434 & -0.10446 & $3.91 \times 10^{-4}$ \\
\hline $\operatorname{cg} 07841173$ & 3 & 128000000 & GATA2 & 0.509397 & 0.616012 & -0.10661 & $2.02 \times 10^{-2}$ \\
\hline $\operatorname{cg} 13274938$ & 17 & 38493822 & RARA & 0.604901 & 0.715056 & -0.11016 & $8.96 \times 10^{-5}$ \\
\hline $\operatorname{cg} 00514241$ & 22 & 28193910 & MN1 & 0.496668 & 0.608136 & -0.11147 & $1.81 \times 10^{-3}$ \\
\hline $\operatorname{cg} 20408276$ & 2 & 38300586 & CYP1B1 & 0.179514 & 0.291561 & -0.11205 & $3.76 \times 10^{-3}$ \\
\hline $\operatorname{cg} 11656478$ & 2 & 38297759 & CYP1B1 & 0.289594 & 0.415179 & -0.12559 & $7.53 \times 10^{-3}$ \\
\hline $\operatorname{cg} 18929894$ & 12 & 14522829 & ATF7IP & 0.661424 & 0.792015 & -0.13059 & $1.20 \times 10^{-4}$ \\
\hline cg20254225 & 2 & 38301438 & CYP1B1 & 0.280009 & 0.412563 & -0.13255 & $7.24 \times 10^{-4}$ \\
\hline $\operatorname{cg} 17514528$ & 1 & 11862907 & MTHFR & 0.352532 & 0.491889 & -0.13936 & $1.18 \times 10^{-4}$ \\
\hline cg02162897 & 2 & 38300537 & CYP1B1 & 0.207392 & 0.348464 & -0.14107 & $3.18 \times 10^{-4}$ \\
\hline $\operatorname{cg} 06264984$ & 2 & 38300885 & CYP1B1 & 0.348033 & 0.512794 & -0.16476 & $4.73 \times 10^{-5}$ \\
\hline cg09799983 & 2 & 38301756 & CYP1B1 & 0.397007 & 0.574734 & -0.17773 & $9.42 \times 10^{-5}$ \\
\hline
\end{tabular}

AML, acute myeloid leukemia; Chr, chromosome; Loc, location.

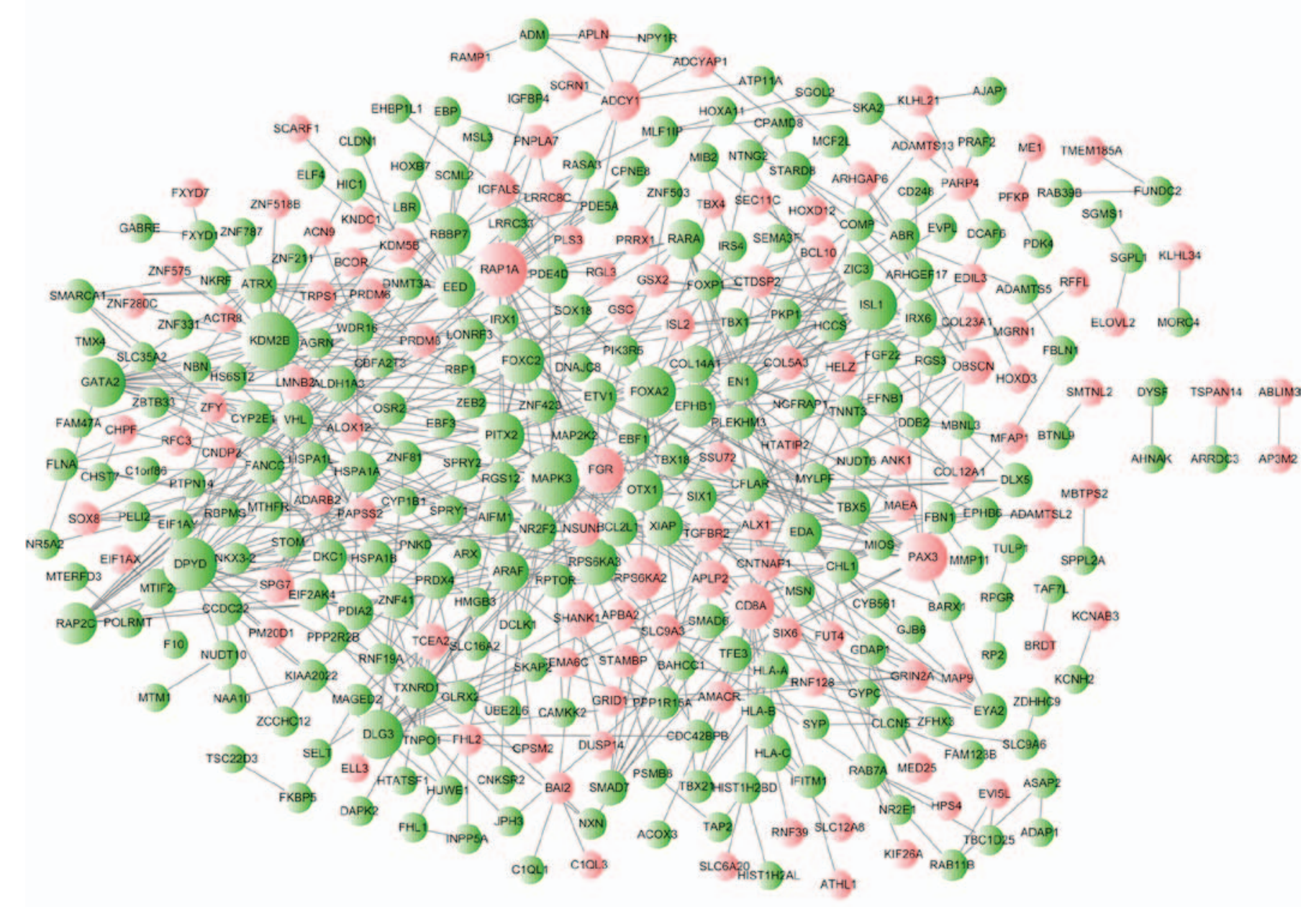

Figure 4. Protein-protein interaction network of aberrantly methylated genes. Node sizes correspond to the degree value. Red nodes indicate hypermethylated genes and green nodes indicate hypomethylated genes. 
genes were derived from the CTD database. Only 11 genes, including $C Y P 1 B 1$, were shown to be differentially methylated in the BM-MSCs from patients with AML in this study. The detailed information on these 11 leukemia-associated differentially methylated genes is summarized in Table III.

\section{Discussion}

In the present study, the methylation status of multiple $\mathrm{CpG}$ sites and $\mathrm{CpG}$ islands was examined in BM-MSCs from a series of AML patients compared with normal BM-MSC from control donors. The results showed that 228 hypermethylated CpG site probes covering 183 gene symbols and 523 hypomethylated $\mathrm{CpG}$ site probes covering 362 gene symbols were identified in the BM-MSCs from the AML patients. Overall, 4 genes with $\mathrm{CpG}$ island hypermethylation were identified, including PM20D1. The hsa-miR-596-encoding gene MIR596 was found to be hypermethylated and the regulation network based on hsa-miR-596 and its targets (such as CYP1B1) was constructed. Hypermethylated and hypomethylated genes were enriched in different KEGG pathways, including 'hsa05221: Acute myeloid leukemia' and 'hsa05220: Chronic myeloid leukemia', which the hypomethylated gene MAPK3 showed involvement in. In addition, $M A P K 3, K D M 2 B$ and RAPIA were hubs in the PPI network of methylated genes.

Cytosine methylation is a DNA modification generally associated with transcriptional silencing (28). It is clear that the hypermethylation of $\mathrm{CpG}$ islands concomitant with global hypomethylation is a feature of nearly all human cancer types (29). When identifying DMRs between cancer genomes of cells or tissues from patients and normal cells or tissues from control donors, a number of the most relevant DNA methylation differences occur at $\mathrm{CpG}$ island regions (30). Detecting hypermethylation of $\mathrm{CpG}$ islands of genes has emerged as a promising method for the diagnosis and monitoring of cancer (29). In the present study, $P M 20 D 1$ was identified with hypermethylated $\mathrm{CpG}$ islands, indicating that the expression of PM20D1 was downregulated or silenced. PM20D1, a secreted enzyme, exhibits hydrolytic and catalytic activity to reversibly form N-acyl amino acids (31). Recently, Long et al (32) reported that the PM20D1 enzyme could tack lipids on to amino acids and that these generated $\mathrm{N}$-acyl amino acids directly activated mitochondria for thermogenesis. Non-shivering thermogenesis is a major component of energy expenditure and has been implicated in the regulation of body weight (33). Moreover, evidence shows that children with acute lymphoblastic leukemia typically gain weight and do so at excessive rates, and are significantly fatter than children with other malignancies and healthy sibling controls (34). In accordance with previous studies, we hypothesized that PM2OD1 with hypermethylation of $\mathrm{CpG}$ islands may be associated with the energy expenditure of patients with AML. However, these issues must now be addressed in further studies that include larger numbers of patients.

Studies have shown that deregulated expression of miRNAs may occur in disease, and methylation has been considered as one of the mechanisms that may be associated with miRNA silencing (35). For instance, the study by Heller et al (35) identified that miR-9-3 and miR-193a were tumor specifically methylated in patients with non-small cell lung cancer, and the methylation of miRNAs could be used as the prognostic parameter. Additionally, another study suggested that miR-596 may be tumor-suppressive miRNA in oral cancer, and DNA hypermethylation of the $\mathrm{CpG}$ island of the miR-596 gene was frequently observed in oral cancer cell lines (36). In the present study, it was found that the hsa-miR-596-encoding gene MIR596 was hypermethylated in the BM-MSCs from a series of AML patients. Furthermore, miR-596 could regulate several hypermethylated and hypomethylated genes in the regulation network. Thus, it was suggested that the hypermethylation of miR-159 may be associated with its transcriptional regulation. It was also inferred that miR-159 methylation in BM-MSCs may be a biomarker or prognostic factor for patients with AML. However, the significance of the methylation of miR-159 demands further investigation.

In conclusion, the present results provide evidence for epigenetic changes and widespread methylation of genes in BM-MSCs from patients with AML. PM20D1 with hypermethylation of $\mathrm{CpG}$ islands may be associated with the energy expenditure of patients with AML. Furthermore, the aberrant hypermethylated miR-159-encoding gene MIR159 may be a potential biomarker of AML. Methylation profiling of larger sample groups may aid in clarifying whether aberrant methylation of these genes in AML is random or specific. However, these findings may provide potential biomarkers and reveal information regarding the pathological mechanism of AML.

\section{Acknowledgements}

The founders supporting this study are listed as follows: The National Natural Science Foundation of China (grant no. 81400168), the Guangzhou Health Care and Cooperative Innovation Major Project (grant no. 201400000003-1), the Science and Technology Planning Project of Guangdong, China (grant nos. 2014A020209047 and 2015A020210068), the Foundation of Guangdong Traditional Chinese Medicine (grant no. 20131104), the Foundation of Guangdong Medicine (grant nos. A2013128 and A2017266), the Foundation of Technological Support of Xinjiang Uygur Autonomous region (grant no. 201491185) and the Foundation of Guangdong Second Provincial General Hospital (grant nos. YY2014-002, YQ2015-004/005/012/016, YQ2016-011/013, 2017-001).

\section{References}

1. Grove CS and Vassiliou GS: Acute myeloid leukaemia: A paradigm for the clonal evolution of cancer? Dis Model Mech 7: 941-951, 2014.

2. Estey EH and Ayalew T: Acute myeloid leukemia: 2012 update on diagnosis, risk stratification, and management. Am J Hematol 87: 89-99, 2012.

3. Ferrara $\mathrm{F}$ and Schiffer $\mathrm{CA}$ : Acute myeloid leukaemia in adults. Lancet 381: 484-495, 2013.

4. Zhou M and Tong X: Downregulated Poly-C binding protein-1 is a novel predictor associated with poor prognosis in Acute Myeloid Leukemia. Diagn Pathol 10: 147, 2015.

5. Kwon HY, Bajaj J, Ito T, Blevins A, Konuma T, Weeks J, Lytle NK, Koechlein CS, Rizzieri D, Chuah C, et al: Tetraspanin 3 is required for the development and propagation of acute myelogenous leukemia. Cell Stem Cell 17: 152-164, 2015.

6. Creutzig U, van den Heuvel-Eibrink MM, Gibson B, Dworzak MN, Adachi S, de Bont E, Harbott J, Hasle H, Johnston D, Kinoshita A, et al; AML Committee of the International BFM Study Group: Diagnosis and management of acute myeloid leukemia in children and adolescents: Recommendations from an international expert panel. Blood 120: 3187-3205, 2012. 
7. Li S, Garrett-Bakelman FE, Chung SS, Sanders MA, Hricik T, Rapaport F, Patel J, Dillon R, Vijay P, Brown AL, et al: Distinct evolution and dynamics of epigenetic and genetic heterogeneity in acute myeloid leukemia. Nat Med 22: 792-799, 2016.

8. Chen C, Liu Y, Lu C, Cross JR, Morris JP IV, Shroff AS, Ward PS, Bradner JE, Thompson C and Lowe SW: Cancer-associated IDH2 mutants drive an acute myeloid leukemia that is susceptible to Brd4 inhibition. Genes Dev 27: 1974-1985, 2013.

9. Figueroa ME, Lugthart S,Li Y,Erpelinck-Verschueren C, Deng X, Christos PJ, Schifano E, Booth J, van Putten W, Skrabanek L, et al: DNA methylation signatures identify biologically distinct subtypes in acute myeloid leukemia. Cancer Cell 17: 13-27, 2010

10. Akalin A, Garrett-Bakelman FE, Kormaksson M, Busuttil J, Zhang L, Khrebtukova I, Milne TA, Huang Y, Biswas D, Hess JL, et al: Base-pair resolution DNA methylation sequencing reveals profoundly divergent epigenetic landscapes in acute myeloid leukemia. PLoS Genet 8: e1002781, 2012.

11. Tao YF, Ni J, Lu J, Wang N, Xiao PF, Zhao WL, Wu D, Pang L, Wang J, Feng $X$ and Pan J: The promoter of miR-663 is hypermethylated in Chinese pediatric acute myeloid leukemia (AML). BMC Med Genet 14: 74, 2013.

12. Rodríguez-Pardo VM, Aristizabal JA, Jaimes D, Quijano SM, de los Reyes I, Herrera MV, Solano J and Vernot JP: Mesenchymal stem cells promote leukaemic cells aberrant phenotype from B-cell acute lymphoblastic leukaemia. Hematol Oncol Stem Cell Ther 6: 89-100, 2013.

13. Blau O, Hofmann WK, Baldus CD, Thiel G, Serbent V, Schümann E, Thiel E and Blau IW: Chromosomal aberrations in bone marrow mesenchymal stroma cells from patients with myelodysplastic syndrome and acute myeloblastic leukemia. Exp Hematol 35: 221-229, 2007

14. Roela RA, Carraro DM, Brentani HP, Kaiano JHL, Simão DF, Guarnieiro R, Lopes LF, Borojevic R and Brentani MM: Gene stage-specific expression in the microenvironment of pediatric myelodysplastic syndromes. Leuk Res 31: 579-589, 2007.

15. Barrett T, Troup DB, Wilhite SE, Ledoux P,Evangelista C, Kim IF, Tomashevsky M, Marshall KA, Phillippy KH, Sherman PM et al: NCBI GEO: Archive for functional genomics data sets 10 years on. Nucleic Acids Res 39: D1005-D1010, 2011.

16. von der Heide EK, Neumann M, Vosberg S, James AR, Schroeder MP, Ortiz-Tanchez J, Isaakidis K, Schlee C, Luther M, Jöhrens K, et al: Molecular alterations in bone marrow mesenchymal stromal cells derived from acute myeloid leukemia patients. Leukemia 31: 1069-1078, 2017.

17. Pérez C, Martínez-Calle N, Martín-Subero JI, Segura V, Delabesse E, Fernandez-Mercado M, Garate L, Alvarez S, Rifon J, Varea S, et al: TET2 mutations are associated with specific 5-methylcytosine and 5-hydroxymethylcytosine profiles in patients with chronic myelomonocytic leukemia. PLoS One 7: e31605, 2012.

18. Bibikova M, Lin Z, Zhou L, Chudin E, Garcia EW, Wu B, Doucet D, Thomas NJ, Wang Y, Vollmer E, et al: High-throughput DNA methylation profiling using universal bead arrays. Genome Res 16: 383-393, 2006.

19. Warden CD, Lee H, Tompkins JD, Li X, Wang C, Riggs AD, Yu H, Jove R and Yuan YC: COHCAP: An integrative genomic pipeline for single-nucleotide resolution DNA methylation analysis. Nucleic Acids Res 41: e117-e117, 2013.

20. Robinson MD, Kahraman A, Law CW, Lindsay H, Nowicka M, Weber LM and Zhou X: Statistical methods for detecting differentially methylated loci and regions. Front Genet 5: 324, 2014

21. Dweep H and Gretz N: miRWalk2.0: A comprehensive atlas of microRNA-target interactions. Nat Methods 12: 697, 2015.

22. Smoot ME, Ono K, Ruscheinski J, Wang PL and Ideker T: Cytoscape 2.8: New features for data integration and network visualization. Bioinformatics 27: 431-432, 2011.
23. Huang W, Sherman BT and Lempicki RA: Systematic and integrative analysis of large gene lists using DAVID bioinformatics resources. Nat Protoc 4: 44-57, 2009.

24. Szklarczyk D, Franceschini A, Wyder S, Forslund K, Heller D, Huerta-Cepas J, Simonovic M, Roth A, Santos A, Tsafou KP, et al: STRING v10: Protein-protein interaction networks, integrated over the tree of life. Nucleic Acids Res 43: D447-D452, 2015.

25. Lopes CT, Franz M, Kazi F, Donaldson SL, Morris Q and Bader GD: Cytoscape Web: An interactive web-based network browser. Bioinformatics 26: 2347-2348, 2010.

26. Davis AP, Murphy CG, Johnson R, Lay JM, Lennon-Hopkins K, Saraceni-Richards C, Sciaky D, King BL, Rosenstein MC, et al: The comparative toxicogenomics database: Update 2013. Nucleic Acids Res 39: 1067-1072, 2011

27. Bauer-Mehren A, Bundschus M, Rautschka M, Mayer MA, Sanz F and Furlong LI: Gene-disease network analysis reveals functional modules in mendelian, complex and environmental diseases. PLoS One 6: e20284, 2011.

28. Schübeler D: Function and information content of DNA methylation. Nature 517: 321-326, 2015

29. Wen L, Li J, Guo H, Liu X, Zheng S, Zhang D, Zhu W, Qu J, Guo L, Du D, et al: Genome-scale detection of hypermethylated $\mathrm{CpG}$ islands in circulating cell-free DNA of hepatocellular carcinoma patients. Cell Res 1376: 25, 2015.

30. Jung M, Kadam S, Xiong W, Rauch TA, Jin SG and Pfeifer GP: MIRA-seq for DNA methylation analysis of $\mathrm{CpG}$ islands. Epigenomics 7: 695-706, 2015.

31. Larrick JW, Larrick JW and Mendelsohn AR: Uncoupling Mitochondrial Respiration for Diabesity. Rejuvenation Res 19 337-340, 2016

32. Long JZ, Svensson KJ, Bateman LA, Lin H, Kamenecka T, Lokurkar IA, Lou J, Rao RR, Chang MR, Jedrychowski MP, et al: The Secreted Enzyme PM20D1 Regulates Lipidated Amino Acid Uncouplers of Mitochondria. Cell 166: 424-435, 2016.

33. Matsuda J, Hosoda K, Itoh H, Son C, Doi K, Tanaka T, Fukunaga Y, Inoue G, Nishimura H, Yoshimasa Y, et al: Cloning of rat uncoupling protein-3 and uncoupling protein-2 cDNAs: Their gene expression in rats fed high-fat diet. FEBS Lett 418 200-204, 1997.

34. Warner JT, Evans WD, Webb DKH and Gregory JW: Body composition of long-term survivors of acute lymphoblastic leukaemia. Med Pediatr Oncol 38: 165-172, 2002.

35. Heller G, Weinzierl M, Noll C, Babinsky V, Ziegler B, Altenberger C, Minichsdorfer C, Lang G, Döme B, End-Pfützenreuter A, et al: Genome-wide miRNA expression profiling identifies miR-9-3 and miR-193a as targets for DNA methylation in non-small cell lung cancers. Clin Cancer Res 18: $1619-1629,2012$

36. Endo H, Muramatsu T, Furuta M, Uzawa N, Pimkhaokham A, Amagasa T, Inazawa $J$ and Kozaki K: Potential of tumor-suppressive miR-596 targeting LGALS3BP as a therapeutic agent in oral cancer. Carcinogenesis 34: 560-569, 2013.

This work is licensed under a Creative Commons Attribution-NonCommercial-NoDerivatives 4.0 International (CC BY-NC-ND 4.0) License. 